PACS 02.70.-c, 02.70.Ns, 61.20.Ja, 82.65.Yh

\title{
STRUCTURE AND DYNAMICS OF WATER AND HYDRATED IONS NEAR A LIQUID MERCURY SURFACE AS STUDIED BY MOLECULAR DYNAMICS SIMULATIONS
}

\author{
Josef Böcker, Gergely Tóth, Karl Heinzinger \\ Max-Planck-Institut für Chemie (Otto-Hahn-Institut), \\ P.O.B. 3060, D-55020 Mainz, Germany
}

Received September 12, 1995

\begin{abstract}
Molecular dynamics simulation studies of pure water and those with either an additional lithium or iodide ion near a liquid mercury surface are presented. The mercury-water, mercury-mercury, mercury-alkali ion, and mercury-halide ion potentials developed recently are introduced. The structure of the interfaces is described by density profiles and radial distribution functions. The spectral densities of the hindered translational motions of water and of both ions, parallel and perpendicular to the surface, are analysed. The simulation results are compared with experimental data of liquid mercury and its liquid-vapour interface.
\end{abstract}

\section{Introduction}

In this presentation we review the results of Molecular Dynamics (MD) simulations of the electrolyte solution-liquid mercury interface achieved so far. In a preceding paper [1] we reported on MD simulations of a rigid mercurywater interface, introducing a mercury-water potential based on the adsorption energy of a water molecule at a mercury cluster [2]. In order to extend these investigations to a liquid mercury-water interface a mercury-mercury potential was recently developed [3], which describes adequately different properties of pure liquid mercury. The atomic densities resulting from the simulations of the interface [4] are in good agreement with the data from an X-ray reflectivity study of the mercury liquid-vapour interface [5]. Additional structural and dynamical properties of the mercury-water interface are analysed.

The mercury-ion potentials for alkali and halide ions were parameterized from $a b$ initio calculations of ion-mercury clusters [6]. Results of MD simulations of the liquid mercury-aqueous solution interface with either one lithium or one iodide ion concern, besides the density profiles of the ions, the influence of the liquid mercury surface on the hydration shell structure of the ions, their influence on the first mercury layer, and the spectral densities of the hindered translational motions of the ions, parallel and perpendicular to the surface [7].

The paper is organized in the following way. The mercury-mercury pair potential, the mercury-water potential, and the mercury-ion potentials are described in Sec. 2.. The simulation procedures are outlined in Sec. 3.. The 
structure of the liquid mercury-pure water interface is discussed in terms of the atomic density profiles perpendicular to the interface and the radial distribution functions for different water and mercury layers in Secs. 4.1. and 4.2., respectively. The rearrangement in both the water and the metal phase induced by an iodide or a lithium ion is discussed in Sec. 4.3.. The dynamical properties of water and the two ions are analysed in Sec. 5.. Finally, the simulation results are summarized in Sec. 6. .

\section{Interaction potentials}

\subsection{Mercury-mercury potential}

The model pseudopotential method [8] was employed to obtain the mercurymercury pair potential. The total potential, which describes the effective interaction between two mercury ions, is given by a sum of an indirect and a direct part

$$
V_{\mathrm{Hg}-\mathrm{Hg}}(r)=V_{\text {ind }}(r)+V_{\text {dir }}(r) .
$$

The potential of indirect interaction between two metal ions, $V_{\text {ind }}(r)$, induced by free electrons, is given in the theory of metals $[8,9]$ by the following equations

$$
\begin{gathered}
V_{\text {ind }}(r)=\frac{\Omega^{2}}{(2 \pi)^{3}} \int_{0}^{\infty} q^{4} w^{2}(q) \frac{1-\epsilon(q)}{\epsilon(q)} \frac{\sin q r}{q r} \mathrm{~d} q, \\
\epsilon(q)=1+\frac{4 \pi}{q^{2}} \Pi(q), \\
\prod(q)=\frac{\prod_{0}(q)}{1-4 \pi q^{-2} G(q) \prod_{0}(q)} \\
\prod_{0}(q)=\frac{k_{\mathrm{F}}}{\pi^{2}}\left(\frac{1}{2}+\frac{4-\eta^{2}}{8 \eta} \ln \left|\frac{2+\eta}{2-\eta}\right|\right), \\
\eta=\frac{q}{k_{\mathrm{F}}}, \quad k_{\mathrm{F}}=\left(\frac{3 \pi^{2} z}{\Omega}\right)^{1 / 3}, \\
G(q)=\frac{1}{2} \frac{\eta^{2}}{\eta^{2}+2\left[1+0.153\left(\pi k_{\mathrm{F}}\right)^{-1}\right]^{-1}} .
\end{gathered}
$$

The following notations are introduced in equations $(2.2-2.7): w(q)$ is the form factor of a bare pseudopotential, $q$ the wave vector, $\epsilon(q)$ the static dielectric function, and $\Pi(q)$ and $\prod_{0}(q)$ are the polarisation operators of the interacting and ideal free electrons, respectively. The local field function $G(q)$ takes into account the correlation effects in the electron subsystem, $z$ is the mercury ion valency and $\Omega$ the volume per ion. The Geldart-Vosko approach [10] is used for $G(q)$, see equation (2.7). The Ashcroft model pseudopotential (MP) with the form factor

$$
w(q)=-\frac{4 \pi z}{\Omega q^{2}} \cos \left(q r_{\mathrm{c}}\right)
$$

and the parameter $r_{\mathrm{c}}$ is employed, see Ref. [8] for details. The energy is written in atomic units. The values $z=2$ a.u., $r_{\mathrm{c}}=0.915$ a.u., and $k_{\mathrm{F}}=$ 0.7091 a.u. determine the indirect ion-ion interaction in liquid mercury [8]. 
The direct potential is given by a sum of a Coulombic and a core overlap contribution (Born-Mayer form), written in atomic units,

$$
V_{\text {dir }}(r)=\frac{z^{2}}{r}+a \exp (-b r) .
$$

The $b$ value was chosen as $1 / r_{c}$. The simulation results [3] of pure liquid mercury with different parameter $a$ values clearly indicated that a very good agreement with experimental data is obtained with an $a$ value of about $2.8 *$ $10^{3} \mathrm{~kJ} / \mathrm{mol}$. In figure 1 the total effective mercury-mercury pair potential $V_{\mathrm{Hg}-\mathrm{Hg}}(r)$, equation (2.1), is shown for $a=2783 \mathrm{~kJ} / \mathrm{mol}$.

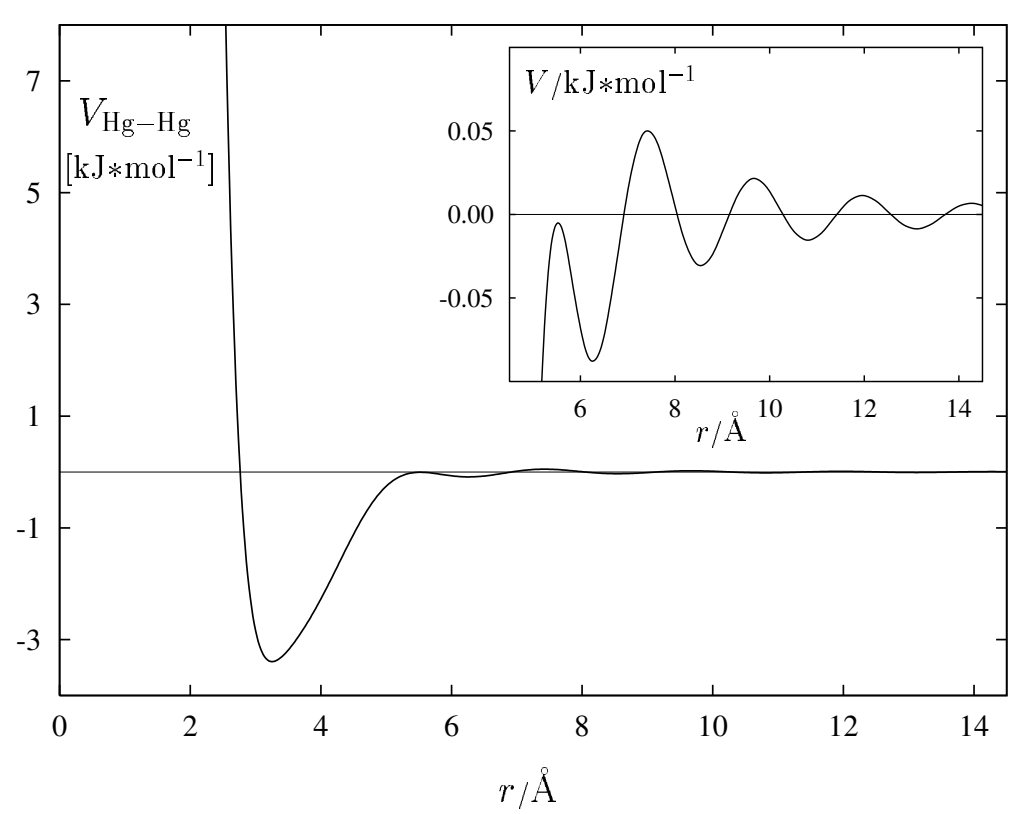

Figure 1. Total effective mercury-mercury pair potential as a function of distance. The inset shows the same function at an expanded scale.

\subsection{Mercury-water potential}

The mercury-water potential was derived from $a b$ initio calculations of the adsorption energy of a water molecule on a planar mercury cluster, consisting of either six or seven atoms [2]. The following potential functions

$$
\begin{gathered}
V_{\mathrm{Hg}-\mathrm{O}}(r, \rho)=[25518 \exp (-2.0829 r)-5508.2 \exp (-1.3922 r)] f(\rho) \\
+8813.2 \exp (-2.1759 r)[1-f(\rho)], \\
V_{\mathrm{Hg}-\mathrm{H}}(r)=2603.6[\exp (-2.2230 r)+\exp (-2.6737 r)], \\
f(\rho)=\exp \left(-0.2213 \rho^{2}\right), \quad \rho=\sqrt{\Delta x^{2}+\Delta y^{2}}
\end{gathered}
$$

yielded the best fit to the $a b$ initio data, where the energies are given in units of $\mathrm{kJ} / \mathrm{mol}$ and the distances in $\AA$. 
The total energy of one water molecule above an infinitely extended rhombohedral mercury surface was calculated for different relative positions. The rhombohedral configuration was found to be a good model for describing the structure of pure liquid mercury [11]. In figure 2 the top, bridge, and hollow sites on a mercury surface are defined. In figure $3 \mathrm{a}$ the potential energy between one water molecule and the rhombohedral mercury surface is shown as a function of the oxygen-surface distance $z$ for the three sites. The water dipole moment is pointing away from the surface. The top site is the energetically most favourable position. The experimental adsorption enthalpy of $-29 \mathrm{~kJ} / \mathrm{mol}$ [12] agrees qualitatively with our result.

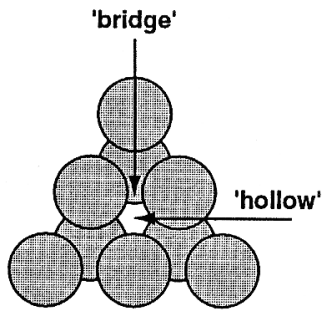

A

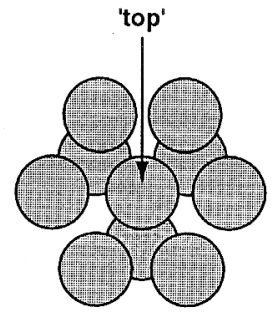

B

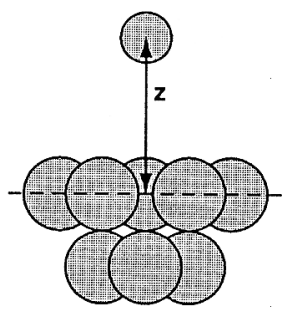

C

Figure 2. Schematic representation of the mercury clusters used in the molecular orbital calculations of the mercuryion interactions. Different adsorption sites, indicated by arrows, are defined. A: The 9 atom cluster used for the bridge and hollow sites. B: The 10 atom cluster used for the top site. C: Definition of the distance $z$ of an ion or an oxygen atom from the surface. The surface plane is indicated by the dashed line.

The total energy was also calculated for different dipole moment orientations. In figure $3 \mathrm{~b}$ the potential energy is shown for the top site with the dipole moment pointing away, parallel, and towards the surface. The parallel orientation is further distinguished by the hydrogen-hydrogen vector being parallel (parallel a) or perpendicular (parallel b) to the surface. The energy minimum is found for an orientation with the dipole moment pointing away from the surface. The energetically most unfavourable situation occurs for an orientation with the dipole moment pointing towards the surface. The same order is found for the bridge and hollow positions.

The overall behaviour of the mercury-water potential is similar to the platinum-water potential discussed in Ref. [13]. The parameterized potential for $\mathrm{Hg}-\mathrm{O}$ and $\mathrm{Hg}-\mathrm{H}$ was employed in an $\mathrm{MD}$ simulation of water near a solid mercury surface [1].

\subsection{Mercury-ion potentials}

All mercury-ion interactions were derived from molecular orbital calculations of one ion and a mercury cluster, consisting of either nine or ten atoms [6]. The clusters are shown in figure 2 together with a schematic representation of the different binding sites. Except for the lithium ion, the interaction potentials are described by the following equations

$$
V_{\mathrm{Hg}-\mathrm{Ion}}(r, \rho)=A \exp (-a r)-B \exp (-b r)+f_{\text {Ion }}(\rho) C \exp (-c r),
$$




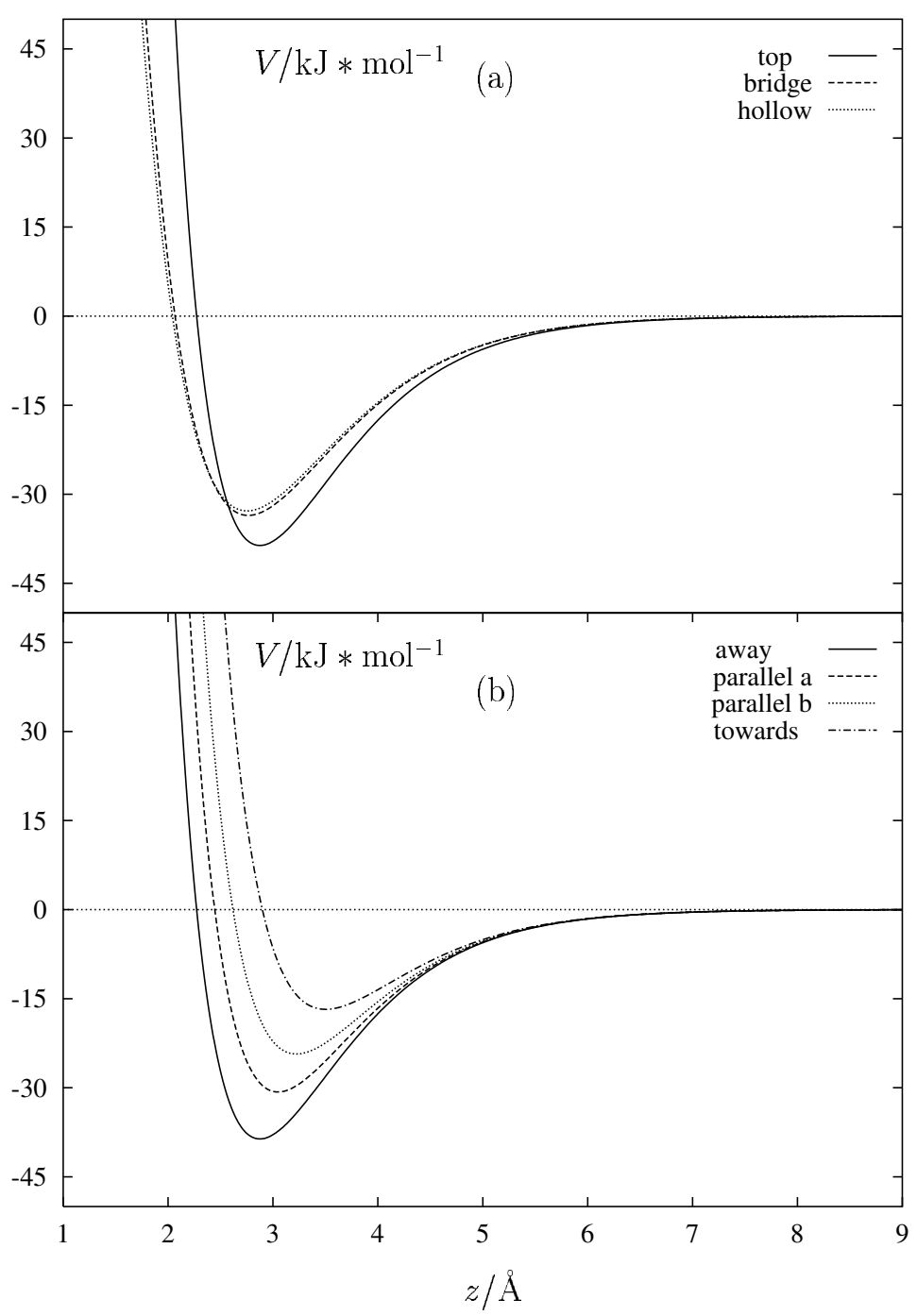

Figure 3. Mercury-water interaction potential as a function of the oxygen-surface distance $z$ for different relative positions and orientations. (a) Top, bridge, and hollow site of a water molecule on the mercury surface with the water dipole moment pointing away from the surface. (b) Top site with the water dipole moment pointing away from, parallel to, and towards the surface. Parallel $\mathrm{a}$ and parallel $\mathrm{b}$ are defined as orientations with the hydrogen-hydrogen vector parallel and perpendicular to the surface, respectively. 
where

$$
f_{\text {Ion }}(\rho)=\exp \left(-d \rho^{p}\right) \text { with } p=(1.52718-\log d) / 0.633954 .
$$

In the case of the mercury-lithium potential, a slightly different analytical form is used

$$
V_{\mathrm{Hg}-\mathrm{Li}}(r, \rho)=A \exp (-a r)-B \exp (-b r)+f_{\mathrm{Ion}}(\rho)[C \exp (-c r)-A \exp (-a r)] .
$$

The constants defining $p$ are chosen in such a way that $f=1$ if $\rho=0$ and $f=0.01$ if $\rho$ corresponds to the nearest mercury atoms of the cluster when the ion is located above the hollow site. The parameters are given in table 2.3.. In figure 4 the potential energies of selected alkali and halide ions are displayed as a function of the distance $z$ from an infinitely extended mercury surface.

Table 1. Mercury-ion potential parameters.

\begin{tabular}{|l|c|c|c|c|c|c|c|}
\hline & $d$ & $A / \mathrm{kJmol}^{-1}$ & $a / \AA^{-1}$ & $B / \mathrm{kJmol}^{-1}$ & $b / \AA^{-1}$ & $C / \mathrm{kJmol}^{-1}$ & $c / \AA^{-1}$ \\
\hline $\mathrm{F}^{-}$ & $1.6139 \cdot 10^{-2}$ & $5.6476 \cdot 10^{5}$ & 4.0420 & $6.2888 \cdot 10^{2}$ & 0.91945 & $-2.0231 \cdot 10^{4}$ & 3.1631 \\
$\mathrm{Cl}^{-}$ & $1.7789 \cdot 10^{-1}$ & $3.3049 \cdot 10^{5}$ & 3.2156 & $3.3957 \cdot 10^{2}$ & 0.78329 & $-2.4884 \cdot 10^{4}$ & 2.8523 \\
$\mathrm{Br}^{-}$ & $1.3530 \cdot 10^{-1}$ & $5.9899 \cdot 10^{5}$ & 3.2909 & $2.3866 \cdot 10^{2}$ & 0.71365 & $-4.7968 \cdot 10^{5}$ & 3.7839 \\
$\mathrm{I}^{-}$ & $9.4896 \cdot 10^{-1}$ & $3.7214 \cdot 10^{5}$ & 2.9385 & $2.0668 \cdot 10^{2}$ & 0.68077 & $-1.4740 \cdot 10^{5}$ & 3.2344 \\
\hline & & & & & & & \\
\hline $\mathrm{Li}^{+}$ & $1.3258 \cdot 10^{-1}$ & $2.7543 \cdot 10^{4}$ & 2.3415 & $1.2613 \cdot 10^{3}$ & 1.07910 & $3.8938 \cdot 10^{4}$ & 2.5451 \\
$\mathrm{Na}^{+}$ & $7.5152 \cdot 10^{-5}$ & $6.6492 \cdot 10^{4}$ & 2.5949 & $5.8475 \cdot 10^{2}$ & 0.93083 & $-7.8324 \cdot 10^{2}$ & 1.9004 \\
$\mathrm{~K}^{+}$ & $8.6680 \cdot 10^{-5}$ & $1.4350 \cdot 10^{5}$ & 2.6167 & $3.6628 \cdot 10^{2}$ & 0.86123 & $-1.0052 \cdot 10^{4}$ & 3.1154 \\
$\mathrm{Rb}^{+}$ & $2.2676 \cdot 10^{-8}$ & $4.0444 \cdot 10^{5}$ & 2.8432 & $1.8858 \cdot 10^{2}$ & 0.74313 & $-9.6312 \cdot 10^{3}$ & 3.0118 \\
$\mathrm{Cs}^{+}$ & $1.2795 \cdot 10^{-5}$ & $5.6117 \cdot 10^{5}$ & 2.7872 & $1.5395 \cdot 10^{2}$ & 0.70905 & $-9.3863 \cdot 10^{3}$ & 3.3035 \\
\hline
\end{tabular}

\subsection{Water-water and water-ion potentials}

The water-water interactions are described by the flexible BJH water model [14]. The lithium-water and the iodide-water potentials employed here are derived again from $a b$ initio calculations [15]. The reader is referred to the references cited for further details of the potentials.

\section{Details of the simulations}

\subsection{Liquid mercury-pure water simulation}

The interface simulation was started from a configuration where the basic periodic boxes from a pure mercury and a pure water simulation were combined at a distance of $3 \AA$, which is approximately the position of the potential minimum for the water-mercury interaction. During the equilibration period the dimensions of the combined box were changed until the average density at the center of the water as well as of the mercury phase showed the density of the pure liquids. The basic box contained 750 water molecules and 880 mercury atoms with sidelengths of $L_{x}=24.00 \AA$, $L_{y}=25.98 \AA$, and $L_{z}=69.80 \AA$ with periodic boundary conditions in all three directions. 


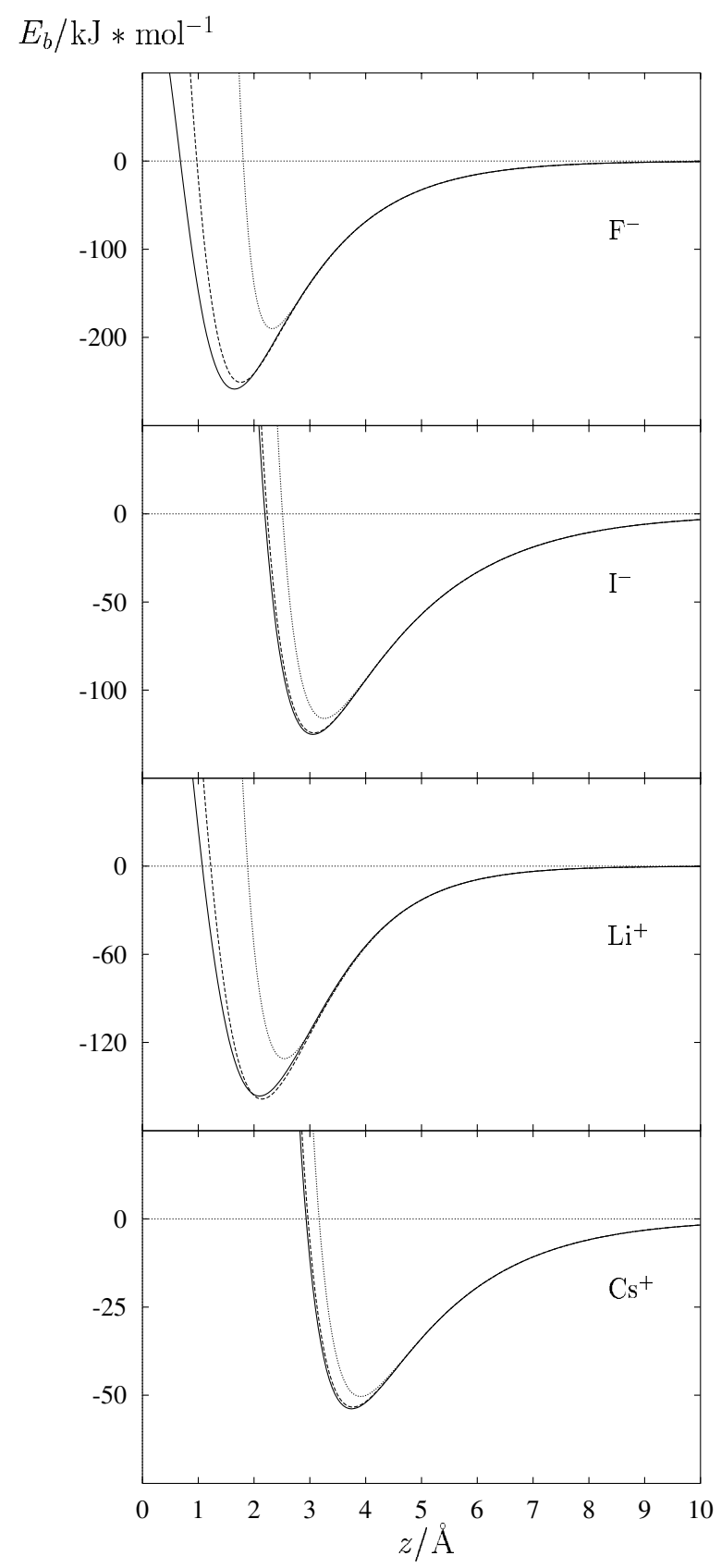

Figure 4. Distance dependence of the binding energy for $\mathrm{F}^{-}, \mathrm{I}^{-}$, $\mathrm{Li}^{+}$, and $\mathrm{Cs}^{+}$calculated from equations (2.13) and (2.15) with the potential parameters given in table 1 for the extended fcc(111) mercury surface. The solid, dashed, and dotted lines denote the hollow, bridge, and topsites, respectively. 
The cutoff radius of $11.96 \AA$ for $V_{\mathrm{Hg}-\mathrm{Hg}}$ was chosen as the position near half the shortest sidelength of the basic box, where the pair potential has a maximum value and the force is therefore zero. A cutoff radius of $11 \AA$ was used for all Coulomb interactions as well as for $V_{\mathrm{Hg}-\mathrm{O}}$ and $V_{\mathrm{Hg}-\mathrm{H}}$. The cutoff radii of the non-Coulombic parts of $V_{\mathrm{O}-\mathrm{O}}, V_{\mathrm{O}-\mathrm{H}}$, and $V_{\mathrm{H}-\mathrm{H}}$ were $9 \AA$, $5 \AA$, and $3 \AA$, respectively. The method of shifted force potentials [18] was employed, except for the $V_{\mathrm{Hg}-\mathrm{O}}$ pair potential.

During the equilibration time of 120 ps we applied temporarily the constant temperature method according to Kast et al. [16]. This method was incorporated into the Adams-Bashford predictor-corrector algorithm [17]. The $N V E$ ensemble without any velocity rescaling was employed during the last 45 ps. The chosen time step of 0.1 fs yielded a relative standard deviation in the total energy of about $1.1 * 10^{-4}$ for $10 \mathrm{ps}$. The last $40 \mathrm{ps}$ were used to analyse the properties of the interface. The average temperature was $299 \mathrm{~K}$.

\subsection{Simulations with ions}

Two molecular dynamics simulations were performed with ions in the $N V T$ ensemble [7]. The basic box contained 400 mercury atoms, 499 water molecules, and one $\mathrm{I}^{-}$or $\mathrm{Li}^{+}$. The simulation cell was rectangular with sidelengths of $L_{x}=L_{y}=19.32 \AA$ and $L_{z}=65.00 \AA$. The arrangement of the water and the metal phase was the same as in the pure water case. The water phase extended over about $40 \AA$ and the metal phase over about $25 \AA$.

The forces were integrated by the fifth order predictor-corrector scheme of Gear. The time step was 0.2 fs. The temperature was held constant at $298 \mathrm{~K}$ by the thermostat of Nosé [19]. Image charge interactions [20] were calculated between the ion image and the water molecules. The other possible image charge interactions were neglected. All interaction potentials were modified using the shifted force method with a cutoff distance of half of the shortest sidelength of the basic box.

An equilibrated liquid mercury-pure water system was used as the starting configuration. The ions were placed in the middle of the water phase. The equilibration of the systems took about $160 \mathrm{ps}$. The $\mathrm{I}^{-}$moved towards the interface during the first $20 \mathrm{ps}$ and then remained in the adsorbed water layer. The $\mathrm{Li}^{+}$stabilized between the third and the fourth water layer for an intermediate time of $60 \mathrm{ps}$. Afterwards, also this ion moved to the interface and remained there. The production runs extended over about 120 ps.

\section{Structure of the interfaces}

\subsection{Density profiles of the liquid mercury-liquid water interface}

The relative density profiles of oxygen, hydrogen, and mercury atoms perpendicular to the mercury surface are displayed in figure 5. The instantaneous average of the $z$ coordinates of the mercury atoms in the mercury layer next to the water phase is used to define $z=0$. The results for the two interfaces are averaged. Both the oxygen and the hydrogen density profiles show two pronounced maxima and a small, third one, indicating a strong layering of water molecules with enhanced densities up to about $8 \AA$. The first, well pronounced oxygen peak with a maximum at $3.0 \AA$ contains $73 \pm 1$ water molecules. The first, strong hydrogen peak at $2.9 \AA$ almost 
coincides with the position of the oxygen one. The small shoulder at $4.0 \AA$ consists of hydrogen atoms which belong to the first adsorbed water layer. These maxima indicate that most water molecules are orientated parallel to the mercury surface, except the ones forming the shoulder at $4.0 \AA$ with an orientation where one hydrogen atom points away from the surface. A second maximum in the oxygen density profile is located at $5.8 \AA$. The density profile of the hydrogen atoms of the second adsorbed water layer is slightly split into a maxima at $6.1 \AA$ and a plateau at $4.9 \AA$. Further analyses show that the hydrogen atoms belonging to the shoulder at $4.0 \AA$ form hydrogen bonds to the oxygen atoms of the second adsorbed water layer and that the hydrogen atoms belonging to the plateau at $4.9 \AA$ form hydrogen bonds to the oxygen atoms of the first adsorbed water layer.

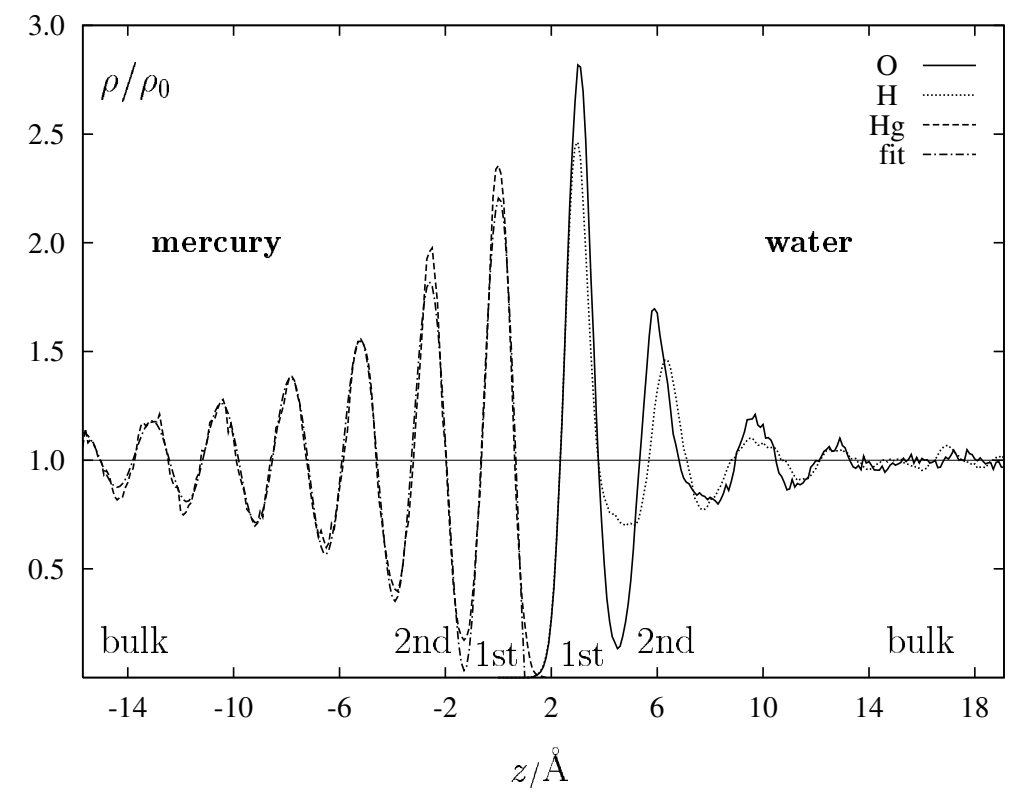

Figure 5. Relative densities of the mercury, oxygen, and hydrogen atoms, derived from the liquid mercury-pure water simulation.

The mercury density profile reveals very long oscillations. It can be represented by the following analytical form

$$
\frac{\rho}{\rho_{0}}(z)=A_{\mathrm{d}}+\alpha_{\mathrm{d}} \exp \left(\beta_{\mathrm{d}} z\right) \cos \left(\gamma_{\mathrm{d}} z\right) .
$$

The best fit is achieved with $A_{\mathrm{d}}=1.013, \alpha_{\mathrm{d}}=1.192, \beta_{\mathrm{d}}=0.150 \AA^{-1}$, and $\gamma_{d}=2.396 \AA^{-1}$. The parameter $A_{d}$ can be taken as a quantitative measure of the mercury density in the center of the mercury phase. This value indicates that the bulk density of mercury is slightly higher than that of pure liquid mercury. The number of mercury atoms in the first layer near the water phase is $72 \pm 1$, whereas the number of the other layers is always $67 \pm 1$. These numbers correspond to relative densities of 1.08 for the first layer and 1.01 for the consecutive layers. Therefore, only the first layer exhibits an increased density, whereas the other layers reveal an average 
density of nearly that of pure liquid mercury. The number of mercury atoms in the first layer is almost identical with the one of oxygen atoms in its first layer, implying that each mercury atom adsorbs one water molecule.

It is interesting to compare the mercury density profile with the one resulting from a recently performed x-ray reflectivity study of the mercury liquid-vapour interface [5]. The authors state that their results provide the only conclusive experimental proof of surface layering in liquid metals to date. The electron density profile of the liquid phase reveals an oscillation length of $2.76 \pm 0.20 \AA$ from the second layer onwards, which is in excellent agreement with our findings of $2.62 \pm 0.04 \AA$. Also their relative densities of the different layers are very similar to our results. The values are $1.09 \pm 0.12$ and $1.08 \pm 0.02$ of the first layer and $1.00 \pm 0.04$ and $1.01 \pm 0.02$ of the other layers, for the x-ray and the MD study, respectively. The first layer of the liquid mercury phase near the vapour phase is much broader [5] compared to the first layer of the mercury phase near the water phase. Another difference between our study and the x-ray one is that a faster decay of the mercury density is observed in the mercury liquid-vapour interface. Obviously, these two differences have to be attributed to the ordering effect of the water phase into the mercury phase.

\subsection{Radial distribution functions at the liquid mercury-liquid water interface}

The mercury-mercury radial distribution function $(\mathrm{RDF}), g_{\mathrm{Hg}-\mathrm{Hg}}(r)$, and the oxygen-oxygen RDF, $g_{\mathrm{O}-\mathrm{O}}(r)$, are shown for different layers in figure $6(\mathrm{a})$ and in figure $6(\mathrm{~b})$, respectively. In order to take account of the restricted volume of a layer the mercury and oxygen densities are not related to a complete spherical volume but to the volume of the specified layer. The layers are defined by two successive minima of the corresponding atomic densities, see figure 5 . The bulk regions for water and mercury are chosen as the regions $12.4<z<19.1$ and $-15.7<z<-10.5 \AA$, respectively. The first maxima of $g_{\mathrm{Hg}-\mathrm{Hg}}(r)$ for the first, second, and bulk layer are positioned at $2.93,3.00$, and $3.00 \AA$, respectively. The value for pure liquid mercury is $3.00 \AA[3,11]$. The first maximum of $g_{\mathrm{O}-\mathrm{O}}(r)$ for the first layer at $2.84 \AA$ does not differ from the value of $2.83 \AA$ for pure water [21]. Therefore, the short range structure of water even in the adsorbed layer mainly reveals the structure of bulk water, obviously to keep the hydrogen bonds.

The $g_{\mathrm{O}-\mathrm{O}}(r)$ for the first layer exhibits a significantly different behaviour in the long range order compared to bulk water, which is visible in the maxima at 5.7, 8.7, and $11.1 \AA$. These maxima reflect the structural arrangement of the first mercury layer, which reveals further maxima at 5.8, 8.6, and $11.3 \AA$. The corresponding maxima for pure mercury are at similar positions of $5.8,8.4$, and $11.0 \AA$ [3]. Overall, the structure of the adsorbed water layer reveals a compromise between keeping the liquid water structure and matching the mercury structure. Further analyses of the directly adsorbed water layer show that most oxygen atoms are located nearly on top of a mercury atom. This position is energetically the most favourable one.

\subsection{Structure of the interface containing ions}

In figure 7 the relative densities of the oxygen, hydrogen, and mercury atoms and those of the ions $-\mathrm{Li}^{+}$or $\mathrm{I}^{-}-$are shown as a function of the distance 


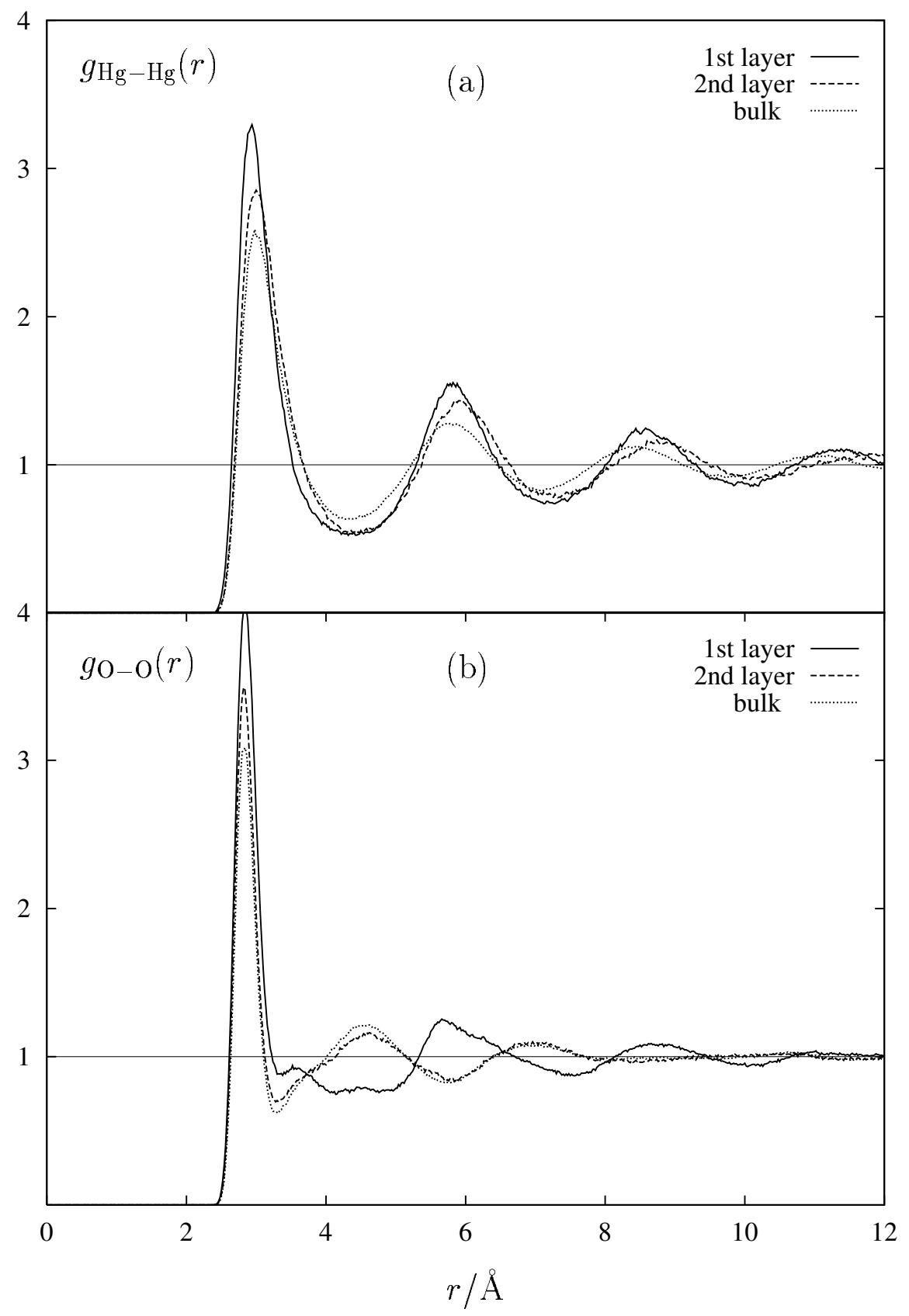

Figure 6. Mercury-mercury (a) and oxygen-oxygen (b) radial distribution functions for different layers, derived from the liquid mercury-pure water simulation. 
from the metal surface on an expanded scale. The positions of the maxima of the density profiles for the two ions almost coincide with those of the oxygen and hydrogen atoms at about $3 \AA$, but for different reasons. The distance of the lithium ion from the surface is solely determined by the $\mathrm{Li}^{+}$-water interaction. In the $\mathrm{I}^{-}$case the ion-metal interaction is responsible for the position of the ion. This is manifested by the total potential energy of the ions, where in the $\mathrm{Li}^{+}$case about $50 \%$ belongs to the ion-water interaction while the rest has to be attributed to the ion-metal interaction. For the $\mathrm{I}^{-}$only about $10 \%$ results from the ion-water interaction. This situation is very similar to that of the $\mathrm{Li}^{+}$and $\mathrm{I}^{-}$near the $\mathrm{Pt}(100)$ surface [15]. There, it was proposed to call the iodide ion contact adsorbed as the ionmetal potential minimum determines the distance of the $\mathrm{I}^{-}$from the surface, while the $\mathrm{Li}^{+}$was not considered contact adsorbed because the $\mathrm{Li}^{+}$-water interaction determines the distance of the ion from the surface, although in both cases no water molecule is found between the ion and the metal surface.

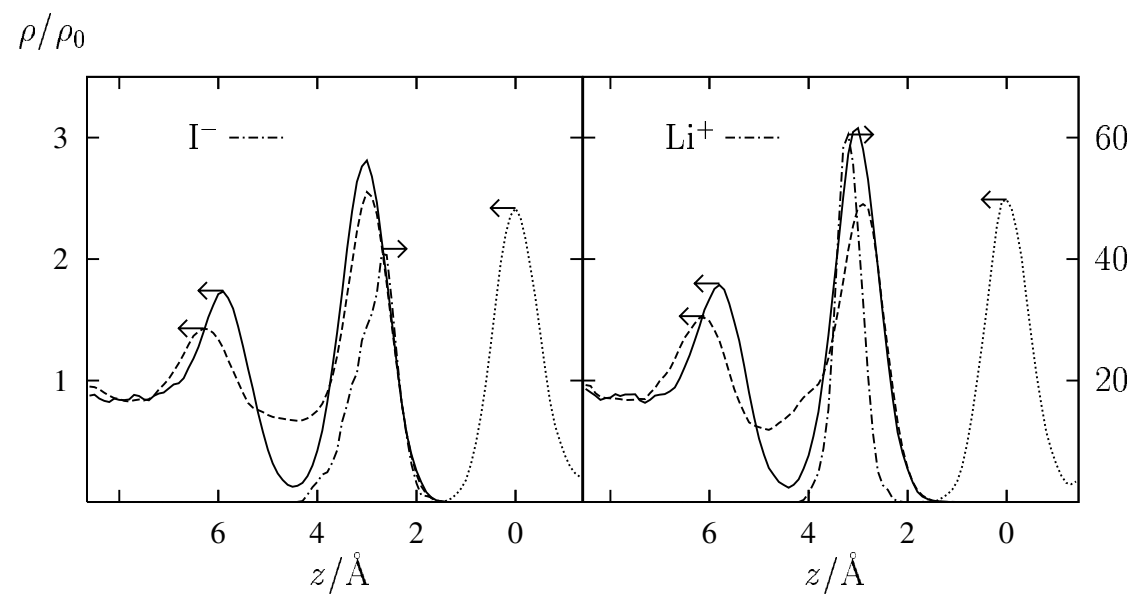

Figure 7. Density profiles for the oxygen (solid), hydrogen (dashed), and mercury (dotted) atoms together with those for $\mathrm{I}^{-}$or $\mathrm{Li}^{+}$(dash-dotted). $z$ denotes the distance from the mercury surface. The arrows indicate the relevant scale.

The ion-oxygen, ion-hydrogen, and ion-mercury RDFs are presented in figure 8 together with the running integration numbers. Both ion-oxygen and ion-hydrogen RDFs are very similar to those in a bulk solution. They are different from those at the Pt(100) surface, where a strongly fixed platinum surface forms a more rigid water layer, which in turn induces longer ranging $\mathrm{RDFs}[15]$. In addition, we show in figure 9 the projections of the trajectories of the surface layer mercury atoms and those of the ions onto the $x y$ plane over a time period of $3 \mathrm{ps,} \mathrm{chosen} \mathrm{arbitrarily} \mathrm{from} \mathrm{the} \mathrm{total}$ simulation time. The trajectories are presented by indicating the $x$ and $y$ values of each particle by a dot after every $0.05 \mathrm{ps}$. The mobile first layer mercury atoms at the liquid mercury surface (see figure 9) adjust in such a way that the ions can keep the hydration structure in the water phase as it exists in the bulk solution. The surface only effects the hydration numbers of the ions by its excluded volume effect. Thus the $\mathrm{Li}^{+}$looses that one of its octahedrally arranged nearest neighbour water molecules which would 


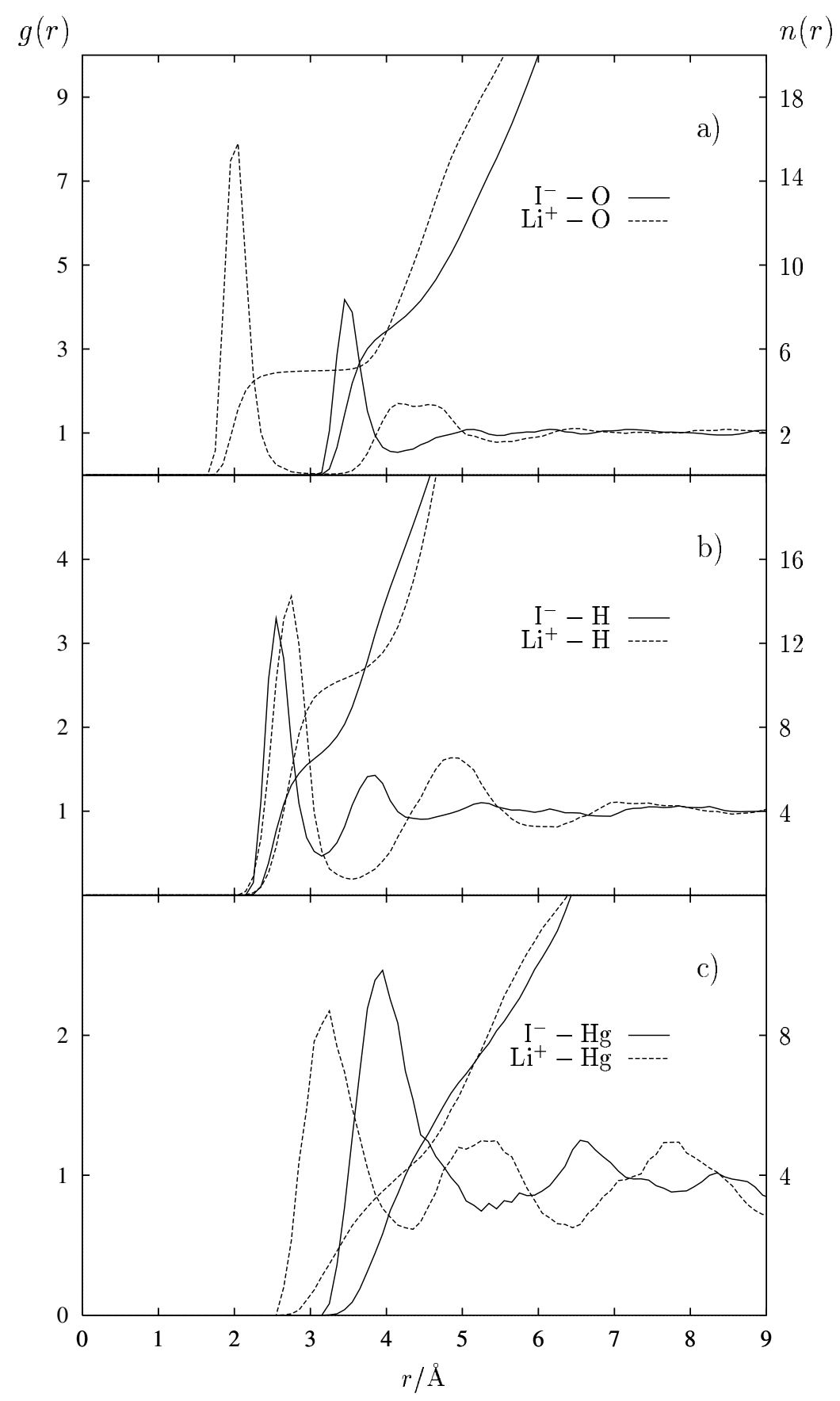

Figure 8. Ion-oxygen (a), ion-hydrogen (b), and ion-mercury (c) radial distribution functions, $g(r)$, and their running integration numbers, $n(r)$. Only the mercury atoms in the first layer are included in the calculations of the ion-mercury RDFs. 


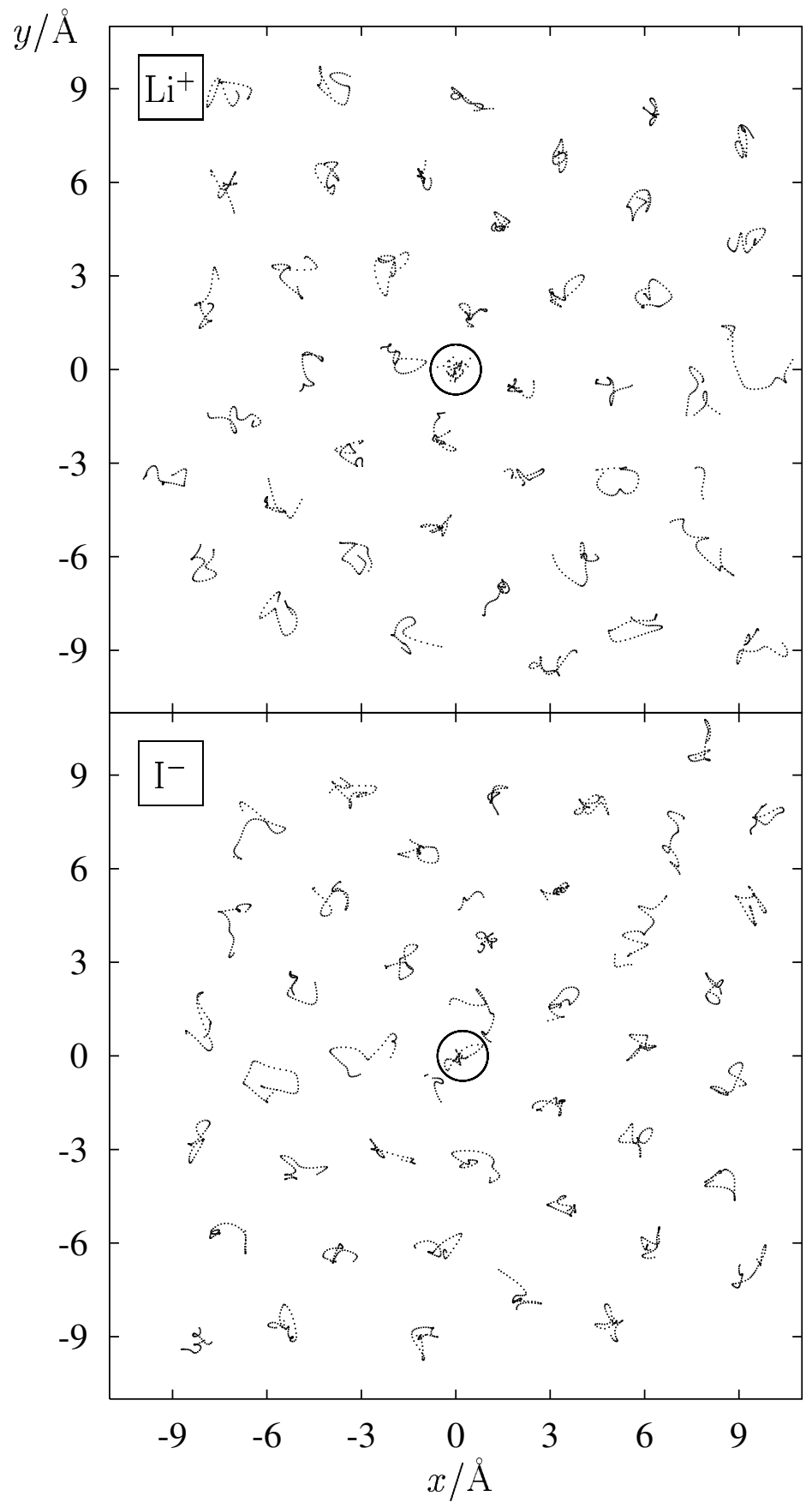

Figure 9. Projections of the trajectories of the ions and the mercury atoms in the first layer onto the $x y$-plane over a 3 ps time interval, chosen arbitrarily from the total simulation time. The positions are marked by a dot after every $0.05 \mathrm{ps}$. The circles enclose the trajectories of the ions. 
be expected between the ion and the mercury surface, just as in the case of the $\operatorname{Pt}(100)$ surface. The number of nearest neighbour water molecules around the $\mathrm{I}^{-}$is reduced in this way to about half of its bulk value. The first hydration shell of the $\mathrm{I}^{-}$shows no symmetry, as in the bulk solution [15].

In the calculations of the ion-mercury RDFs only the first layer mercury atoms are included in order to get information on the structure of the surface layer not disturbed by contributions from inner layers. Both RDFs show significantly broader first maxima and a farther ranging structural order than the ion- $\mathrm{O}$ and ion- $\mathrm{H}$ ones. A longer range order in the ion-mercury RDFs is recognized by strongly pronounced second and third maxima and minima. This long range order is less pronounced for the $\mathrm{I}^{-}$because of its larger surface mobility compared with the $\mathrm{Li}^{+}$(see figure 9).

In order to understand the ion-mercury RDFs and their relationship with the ion density profiles presented in figure 7 we have calculated the average value of the difference in the distances $\Delta z(\rho)=\left\langle z_{\text {ion }}-z_{\mathrm{Hg}}(\rho)\right\rangle$ between the $z$ value of the ion and that of a mercury atom in the surface layer for a given value of $\rho$ (equation 2.12). The result is shown in figure 10 .

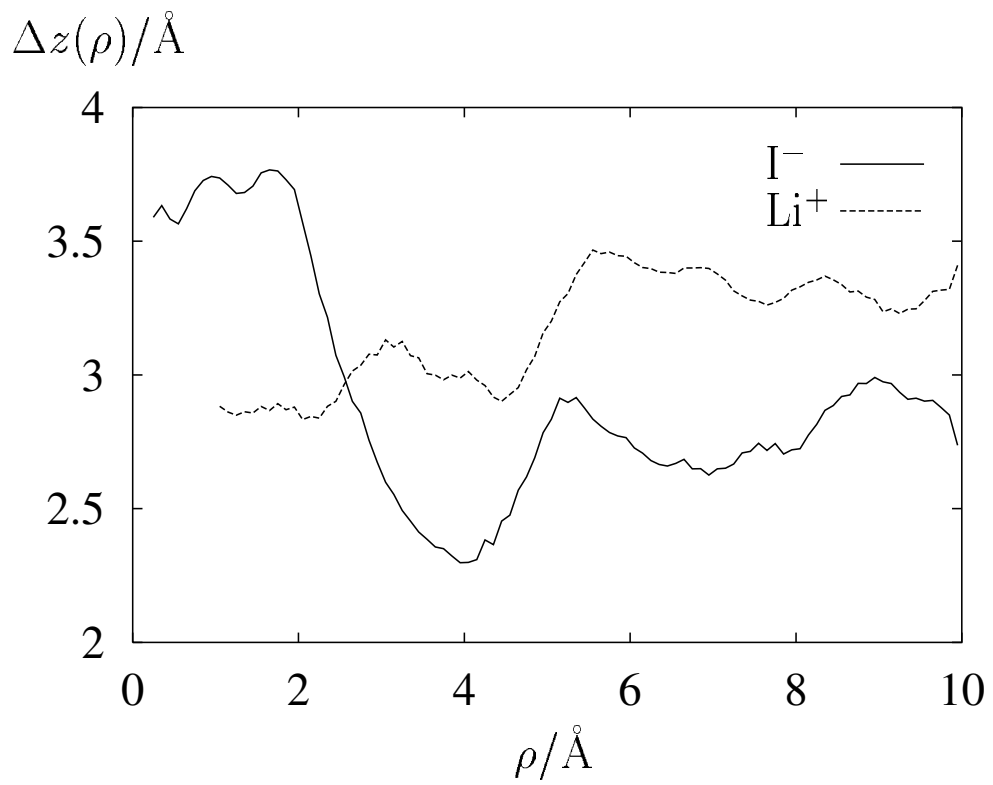

Figure 10. The average value of the difference between the $z$ values of the ions and those of the mercury atoms in the first layer, $\Delta z(\rho)=\left\langle z_{\text {ion }}-z_{\mathrm{Hg}}(\rho)\right\rangle$, as a function of $\rho$, the length of the projection of the distance vector between the ion and a mercury atom onto the $x y$-plane.

The distances $\Delta z(\rho)$ for large $\rho$ (see figure 10) have the highest weight and, therefore, represent the average distance of the ion from the surface, leading to $z$ values of about 3.3 and $2.8 \AA$ for $\mathrm{Li}^{+}$and $\mathrm{I}^{-}$, respectively, in accordance with the maxima in the density profiles of the ions in figure 7 . The first maxima in the ion-mercury RDFs - to be expected at slightly larger $r$ values than the $z$ positions of the ion maxima in the density profiles - can be found at distances of about 3 and $4 \AA$ for $\mathrm{Li}^{+}$and $\mathrm{I}^{-}$, respectively. 
The explanation for even the reversed order of the peak positions can be found in figure 10. For small values of $\rho$ the $\Delta z$ values for $\mathrm{Li}^{+}$and $\mathrm{I}^{-}$are about 2.8 and $3.7 \AA$, respectively. This means that the mercury atoms near the iodide ion are pushed back from the surface significantly and the second nearest neighbours pulled out forming some kind of a small impact crater. The first as well as the second nearest mercury atoms near the lithium ion are pulled out somewhat forming a small hill at the surface. The positions of the first maxima in the RDFs are found at slightly larger distances than those read from figure 10 for small $\rho$ because the ions are not positioned on top of the nearest mercury atom as can be seen from the projections of the trajectories onto the $x y$ plane in figure 9.

The projections of the mercury atom trajectories onto the $x y$ plane (figure 9) cover areas of several $\AA^{2}$ in a time period of $3 \mathrm{ps}$. There appears to be no exchange between positions. Figure 9 shows only a 3 ps section of the trajectories, which seems to be representative for the whole simulation run. After about 5 ps an exchange of mercury atoms between the first and the second layer (in $z$ direction) is observed. From a potential point of view there is practically no difference whether both ions are positioned at a hollow or a bridge site [6].

The fourfold hollow site preferentially occupied by $\mathrm{Li}^{+}$results from the fact that the oxygen atoms on top of the flexible mercury atoms can adjust easily to a distance of $4 \AA$, the diagonal distance between two mercury atoms in a quadratic arrangement which is very favourable for $\mathrm{a} \mathrm{Li}^{+}$-oxygen first neighbour distance of $2 \AA$ in the bulk. The $\mathrm{I}^{-}$cannot occupy a fourfold hollow site because then the four neighbouring mercury atoms would have to be free from water molecules because of the size of the ion, which would be energetically very unfavourable. Therefore, a bridge site is preferentially occupied with only two mercury atoms without water molecules on top (figure 9). The resulting ion positions are quite similar to those in the boundary layer of the $\mathrm{Pt}(100)$ surface. Although the structures of the two surfaces are quite different the flexibility of the liquid mercury surface allows the ions to overcome the structural differences.

\section{Dynamical results}

In order to investigate the hindered translational motions the velocity autocorrelation functions (ACFs) are calculated from the simulations according to

$$
\langle\vec{v}(0) \cdot \vec{v}(t)\rangle=\frac{1}{N_{T} N} \sum_{i=1}^{N_{T}} \sum_{j=1}^{N} \vec{v}_{j}\left(t_{i}\right) \cdot \vec{v}_{j}\left(t_{i}+t\right),
$$

where $N$ denotes the number of particles, $N_{T}$ the number of time averages, and $\vec{v}_{j}(t)$ the velocity of particle $j$ at time $t$. The power spectra are obtained from these ACFs by Fourier transformation. Different from isotropic bulk systems the ACFs of the particles in the lamella are functions of the angle between the velocity vector and the normal vector. Therefore, they are calculated separately for the motions parallel and perpendicular to the surface.

In figure 11 the power spectra of water for different layers and different motions relative to the surface are shown. The first water layer is significantly different for both motions compared to bulk water, whereas the second layer already reveals features similar to bulk water. The principal maximum at $125 \mathrm{~cm}^{-1}$ for the motions perpendicular to the surface is attributed to the mercury-oxygen stretching frequency. This value can also 


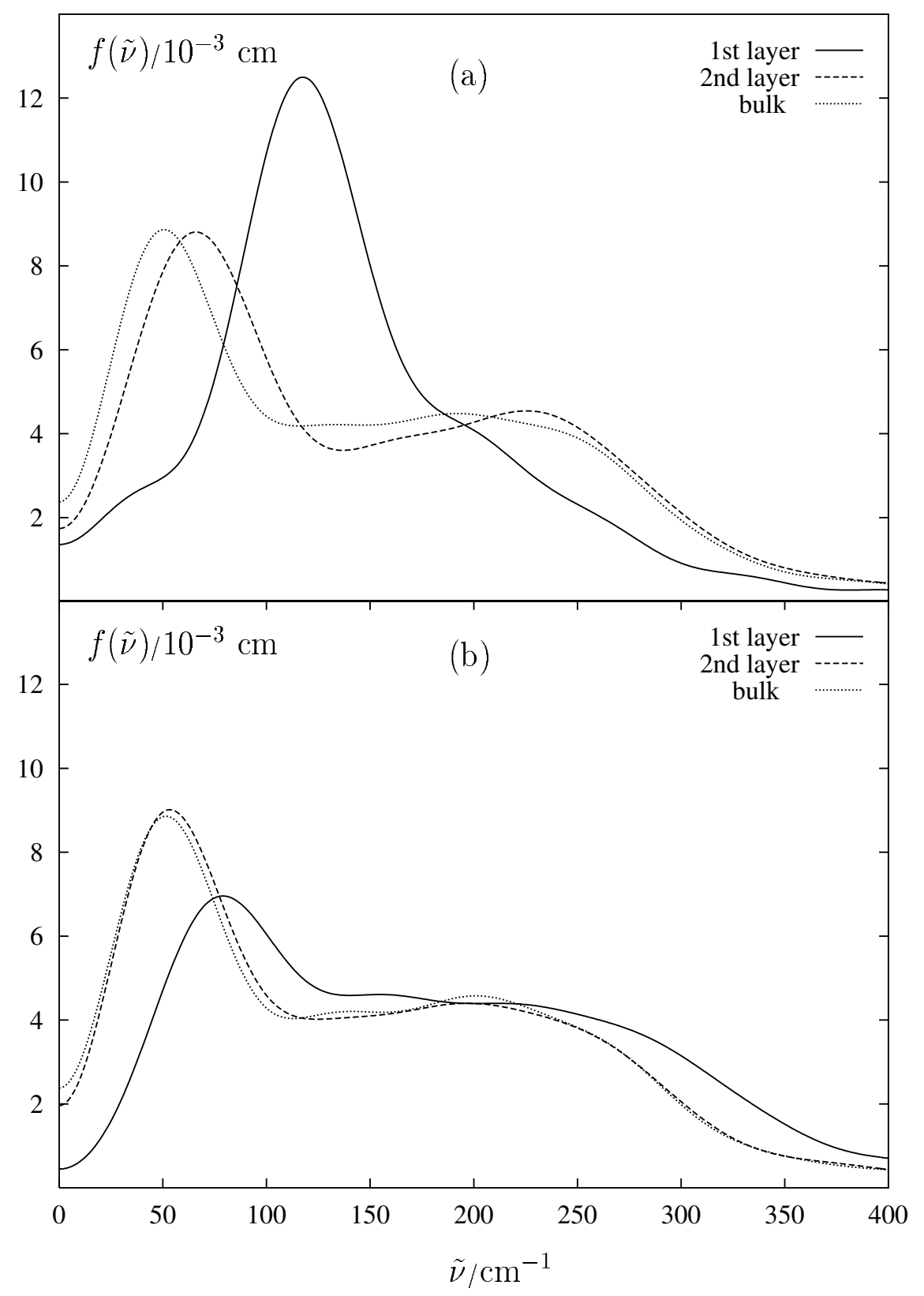

Figure 11. Normalized spectral densities of the hindered translational motions of the water molecules perpendicular (a) and parallel (b) to the surface for different layers, derived from the liquid mercury-pure water simulation. 
be obtained from the force constant of the harmonic oscillator, calculated from the $r$ dependent mercury-water potential energy curve. The contributions from the $\mathrm{O}-\mathrm{O}-\mathrm{O}$ bending and $\mathrm{O}-\mathrm{O}$ stretching motions in bulk water (assigned to the maximum at $50 \mathrm{~cm}^{-1}$ and the shoulder at $200 \mathrm{~cm}^{-1}$, respectively) almost disappear at the expense of the mercury-water interactions. The shoulder at $25 \mathrm{~cm}^{-1}$ is similar to the mercury-mercury stretching frequency, found at $20 \mathrm{~cm}^{-1}$ for pure liquid mercury [3]. A blue shift, from 50 to $80 \mathrm{~cm}^{-1}$, is also visible in the motion of the water molecules parallel to the surface. This indicates that the surface layer is less mobile than bulk water.

The power spectra of the $\mathrm{Li}^{+}$and the $\mathrm{I}^{-}$in the adsorbed layer are depicted in figure 12 . The motions of the $\mathrm{Li}^{+}$in the boundary layer parallel and perpendicular to the metal surface are quite different from those in the isotropic solution, where the maximum is found at about $400 \mathrm{~cm}^{-1}[22]$. The frequencies of the hindered translational motions parallel to the surface show a strong blueshift. This reflects the enhanced $\mathrm{Li}^{+}$-water interactions as a result of the reduced water mobility, which is a consequence of the formation of the water overlayer. The motions of the $\mathrm{Li}^{+}$perpendicular to the surface are characterized by a shift of the main peak in the spectral density to lower frequencies. This is a consequence of the greater mobility of the $\mathrm{Li}^{+}$because of the missing water molecule between the ion and the surface. The positions of the maxima in the spectral densities of the hindered translational motions of the $\mathrm{I}^{-}$differ only slightly for the motions parallel and perpendicular to the surface. These differences are hardly outside the limits of the statistical uncertainty of the calculations. Both are similar to that in the bulk solution, found at about $35 \mathrm{~cm}^{-1}[22]$.
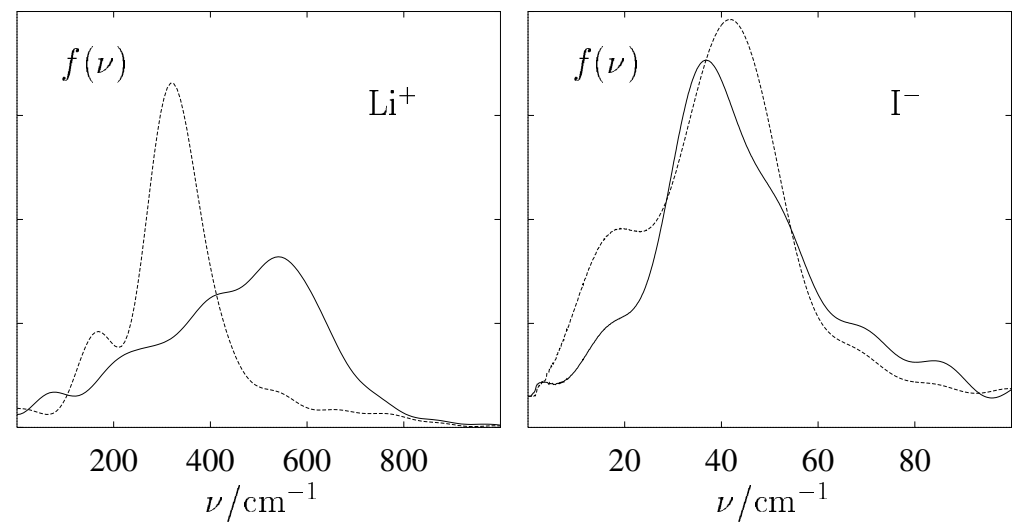

Figure 12. Normalized spectral densities of the hindered translational motions of the lithium and the iodide ions in the boundary layer of the liquid mercury interface calculated separately for the motions parallel (full) and perpendicular (dashed) to the surface.

\section{Summary}

The mercury-water, mercury-mercury, mercury-alkali ion, and mercuryhalide ion potentials developed recently are summarized. Molecular dy- 
namics simulation studies of pure water and those with either an additional lithium or iodide ion near a liquid mercury surface are presented. The resulting mercury density profile reveals very long oscillations and the water density profile is influenced by the metal surface up to a distance of about $8 \AA$. The bulk densities of both mercury and water are almost identical with the ones of the pure liquids. The electron density of the liquid mercury phase, obtained by an x-ray reflectivity study of the mercury liquid-vapour interface, shows an oscillation length in excellent agreement with our findings. Also, the densities of the different layers resulting from the simulations are confirmed by the experiment. Only the adsorbed water layer is significantly different in both structure and dynamics compared with pure water. The structure of the adsorbed water layer reveals a compromise between keeping the short range liquid water structure and matching the long range mercury structure. The hindered translational motions of water perpendicular and parallel to the surface indicate that the adsorbed water layer is less mobile than bulk water.

The flexibility of the mercury phase due to the mercury-mercury potential allows us to analyse the rearrangement induced by either an iodide or a lithium ion in both the water and the metal phase. Both ions adsorb at the mercury surface. The hydration shell structure of the ions is not significantly altered by the mercury surface. The analyses of the hindered translational motions indicate that the lithium ion is less mobile parallel to the surface and more mobile perpendicular to the surface compared to a bulk solution. The iodide ion shows similar dynamics at the interface as in a bulk solution.

\section{References}

[1] Böcker J., Nazmutdinov R.R., Spohr E., Heinzinger K. Molecular dynamics simulation studies of the mercury-water interface. // Surf. Sci., 1995, vol. 335, p. $372-377$.

[2] Nazmutdinov R.R., Probst M., Heinzinger K. Quantum chemical study of the adsorption of an $\mathrm{H}_{2} \mathrm{O}$ molecule on an uncharged mercury surface. // J. Electroanal. Chem., 1994, vol. 369, p. 227-231.

[3] Böcker J., Gurskii Z., Heinzinger K. (to be published).

[4] Böcker J., Spohr E., Heinzinger K. Density profiles at a water/liquid mercury interface. // Z. Naturforsch., 1995, vol. 50a, p. 611-612.

[5] Magnussen O.M., Ocko B.M., Regan M.J., Penanen K., Pershan P.S., Deutsch M. X-ray reflectivity measurements of surface layering in liquid mercury. // Phys. Rev. Lett., 1995, vol. 74, p. 4444-4447.

[6] Tóth G., Spohr E., Heinzinger K. SCF calculations of the interactions of alkali and halide ions with the mercury surface. // Chem. Phys., 1995, vol. 200, p. $347-355$.

[7] Tóth G., Heinzinger K. Molecular dynamics study of an iodide and a lithium ion at the water-liquid mercury interface. // Chem. Phys. Lett., 1995, vol. 245, p. $48-53$.

[8] Ehrenreich H., Seintz F., Turnbull D. Solid state physics. New York/London, Academic Press Inc., 1970, vol. 24.

[9] Harrison W.A. Pseudopotentials in the theory of metals. Benjamin, 1966.

[10] Geldart D.J.W., Vosko S.H. The screening function of an interacting electron gas. // Can. J. Phys., 1966, vol. 44, p. 2137-2171.

[11] Bosio L., Cortes R., Segaud C. X-ray diffraction study of liquid mercury over temperature range 173 to 473 K. // J. Chem. Phys., 1979, vol. 71, p. 35953600 .

[12] Wilkinson M.C. Surface Properties of mercury. // Chem. Rev., 1972, vol. 72, p. $575-625$. 
[13] Spohr E. Computer simulation of the water/platinum interface. // J. Phys. Chem., 1989, vol. 93, p. 6171-6180.

[14] Bopp P., Jancsó G., Heinzinger K. An improved potential for non-rigid water molecules in the liquid phase. // Chem. Phys. Lett., 1983, vol. 98, p. 129-133.

[15] Seitz-Beywl J., Poxleitner M., Heinzinger K. A molecular dynamics study of ionic hydration near a platinum surface. // Z. Naturforsch., 1991, vol. 46a, p. $876-886$.

[16] Kast S.M., Nicklas K., Bär H.-J., Brickmann J. Constant temperature molecular dynamics simulations by means of a stochastic collision model. I. Noninteracting particles. // J. Chem. Phys., 1994, vol. 100, p. 566-576.

[17] Hammimg R.W. Numerical Methods for Scientists and Engineers. New York, McGraw-Hill, 1962.

[18] Streett W.B., Tildesley D.J., Saville G. Multiple time step methods and an improved potential function for molecular dynamics simulations of molecular liquids. In: Computer modelling of matter (ed. Lykos P.). ACS Symp. Ser. Washington, American Chemical Society, 1978, vol. 86.

[19] Nosé S. A unified formulation of the constant temperature molecular dynamics methods. // J. Chem. Phys., 1984, vol. 81, p. 511-519.

[20] Spohr E., Heinzinger K. Molecular dynamics simulation of a water/metal interface. // Chem. Phys. Lett., 1986, vol. 123, p. 218-221.

[21] Pálinkás G., Hawlicka E., Heinzinger K. Molecular dynamics simulations of water-methanol mixtures. // Chem. Phys., 1991, vol. 158, p. 65-76.

[22] Heinzinger K., Seitz-Beywl J., Poxleitner M. Microscopic models of electrodeelectrolyte interfaces (eds. Halley J. W., Blum L.). Electrochem. Soc. Proceedings Series, 1993, vol. 93-5.

\title{
ДОСЛІДЖЕННЯ СТРУКТУРИ ТА ДИНАМІКИ ВОДИ Й ГІДРАТОВАНИХ ІОНІВ ПОБЛИЗУ ПОВЕРХНІ РІДИНА-РТУТЬ МЕТОДОМ МОЛЕКУЛЯРНОӦ ДИНАМІКИ
}

\author{
Й.Бокер, Г.Тот, К.Хайнцінгер
}

\begin{abstract}
Представлені результати молекулярної динаміки дослідження чистої води й такої, що має додатково в собі літій або йод поблизу поверхні рідина-ртуть. Представлено недавно отримані потенціали ртуть-вода, ртуть-ртуть, ртуть-іон-алкалоїд та ртуть-іон галогену. Структура поверхнонь описується профілями густини та радіальними функціями розподілу. Аналізуються спектральні густини перешкоджаючого трансляційного руху води та обох сортів іонів паралельно та перпендикулярно до поверхні. Результати розрахунків порівнюються з експериментальними даними для рідкої ртуті та їі межі поділу рідина-пара.
\end{abstract}

